
\title{
25 Research Suare \\ Oxygen Uptake Response to Switching Stairs Exercise by Non-parametric Modeling
}

Hairong Yu

Sun Yat-sen University

Tian Xie

Sun Yat-sen University

Steven Su ( $\nabla$ steven.su@uts.edu.au )

University of Technology Sydney

Article

Keywords:

Posted Date: May 2nd, 2022

DOI: https://doi.org/10.21203/rs.3.rs-1391204/v2

License: (c) (1) This work is licensed under a Creative Commons Attribution 4.0 International License. Read Full License 


\title{
Oxygen Uptake Response to Switching Stairs Exercise by Non-parametric Modeling
}

\author{
Hairong $\mathrm{Yu}^{1}$, Tian $\mathrm{Xie}^{1}$, and Steven $\mathrm{Su}^{2, *}$ \\ ${ }^{1}$ Guangdong Provincial Engineering and Technology Center of Advanced and Portable Medical Devices, School of \\ Biomedical Engineering, Sun Yat-sen University, Guangzhou, 510006, China \\ ${ }^{2}$ School of Biomedical Engineering, University of Technology Sydney, Sydney, 2007, Australia \\ *steven.su@uts.edu.au
}

\begin{abstract}
Oxygen uptake plays a vital role in evaluating endurance performance during exercise and is widely used for metabolic assessment. In this study, the oxygen uptake during the exercise phase (i.e. ascending or descending) of the stairs exercise are recorded and the experimental dataset features ten participants and a range of different exercise periods. Based on the designed experiment protocol, a non-parametric modeling method with kernel-based regularization is generally applied to estimate the oxygen uptake changes during the switching stairs exercise that is close to the daily life status. Compared with the fixed-order models on accuracy, stability and compatibility, the modeling results demonstrate the effectiveness of the non-parametric modeling approach. The influence of exercise duration on estimated fitness reveals that the model of the phase-oxygen uptake system is not time-invariant related to respiratory metabolism regulation and muscle fatigue. Accordingly, it allows us to study the humans' conversion mechanism at different metabolic rates and facilitates the standardization and development of exercise prescriptions.
\end{abstract}

\section{Introduction}

As a part of the medical paradigm for disease prevention and treatment, exercise prescription is widely used in clinical medicine. The most commonly used aerobic exercise is a type of exercise performed at a moderate level of intensity for an extended period of time that can improve cardiopulmonary function, which is not only one of the most important characteristics of the whole body but also facilitates muscle energetics during physical activity ${ }^{123}$. Among the various kinds of aerobic exercise, the interval training exercise, e.g., the ascending-descending switching exercise, has been confirmed as an effective protocol to enhance cardiovascular fitness and prolong life ${ }^{4}$. The portable sensor can conveniently measure the heart rate (HR) of participants to ensure that the exercise is conducted under aerobic conditions ${ }^{567}$. The respiratory information, such as voluntary ventilation, oxygen uptake $\left(\dot{V} \mathrm{O}_{2}\right)$ or carbon dioxide output $\left(\dot{V} \mathrm{CO}_{2}\right)$, is also commonly used to assess the metabolism demands ${ }^{89101112}$. Aerobic metabolism is a key determinant of endurance performance during exercise ${ }^{13}$. The ability to maintain exercise mainly depends on the participants' capacity to deliver and utilize oxygen at a rate commensurate with energy requirements, which can be characterized by $\dot{V} \mathrm{O}_{2}{ }^{11}$. Therefore, evaluating respiratory information during exercise is vital for the assessment of activities of daily living (ADL).

For the past four decades, the respiratory information, such as $\dot{V} \mathrm{O}_{2}$ and $\dot{V} C \mathrm{O}_{2}$, have been measured at various sports laboratories by the means of the gas analyzer ${ }^{1415}$. The HR, $\dot{V} O_{2}$ and $\dot{V} C O_{2}$ keeps increasing until participants reach the peak level in terms of going upstairs (ascending). When going downstairs (descending), these figures decrease to form a valley ${ }^{5}$. Since the intensity of the stairs exercise can be adjusted by the exercise phase (ascending or descending) without causing any discomfort to the participants, the regular stairs exercise is a proper method to investigate the cardiopulmonary response to the interval training exercise. Due to the difficulties of practically measuring $\dot{V} O_{2}$ in free living, different methods to estimate $\dot{V} O_{2}$ using wearable sensors have been developed ${ }^{16}$. The ascending or descending phase can be considered as a square signal if we describe ascending and descending as two numerical indicators. The above physiological signals, such as $\mathrm{HR}$ and $\dot{V} \mathrm{O}_{2}$, change with the phase. This means that the human cardiovascular system can be described as a dynamic model wherein the input is the phase and the output is the physiological signal. Proper modeling of the interval training exercise is necessary in order to provide an accurate $\dot{V} \mathrm{O}_{2}$ estimation.

One of the challenges in relation to the modeling of the human physiological variable is the fact that the stimulation of the input is often limited. This prevents the use of models with high-dimensional parameters, as they usually lead to ill-posed inverse problems. Recently, the intrinsical ill-posed problem has been circumvented with kernel-based regularization methods, which also admit a Bayesian interpretation. In particular, a non-parametric modeling approach is proposed, in which the impulse response is modeled as a zero-mean Gaussian process. In this way, prior information is introduced in the identification 
process by assigning a kernel covariance ${ }^{171819}$. When the structure of the system cannot be determined or it is too complicated to be described by a simple parametric model, the non-parametric modeling method is the preferable choice ${ }^{2021}$. Furthermore, the development of wearable equipment, such as the $K 4 b^{2}$, makes it possible to record large amounts of data compared to the limited amount of data that was recorded in the past. The traditional modeling method achieves good fitness with a limited amount of data, while the non-parametric model accommodates a large dataset. The non-parametric model with the kernel-based regularization approach has been applied by several researchers to system identification with different demands ${ }^{222324}$. This has achieved high accuracy and robust system identification when it has been applied to the dynamics of physiological information responses during the exercise phase within a well-designed kernel strategy and a regularization term.

In this paper, we propose a novel $\dot{V} \mathrm{O}_{2}$ estimation method based on a non-parametric model to investigate the dynamics and variability of the human body's physiological response to an interval training exercise. Our study provides the following contributions: (1) We introduce a stable and compatible method to accurately estimate the $\dot{V} \mathrm{O}_{2}$ during the continuously changing stairs exercise. Using the non-parametric model allows for the prediction of human respiratory system responses due to its potential applicability for the regulation of oxygen uptake during interval stair training exercise. Then, different types of fixed-order models are employed for comparison purposes. (2) We evaluate the dynamic $\dot{V} \mathrm{O}_{2}$ response during the different periods of stairs exercise. The fitness of models decreases with increases to the exercise period indicating the changing system structure, which is related to the modulation of respiratory metabolism and muscle fatigue. The continuous and random changes in the respiratory system response to the exercise phase should be considered in the formulation of exercise prescriptions.

The remainder of this paper is organized as follows. Section introduces the non-parametric model with the kernelbased regularization. This includes, the experiment, the pre-processing, and the verification approach. Section presents the identification and comparison results. Section analyzes and discusses the results. Finally, a conclusion is drawn in Section .

\section{Methods}

All methods were performed in accordance with the relevant guidelines and regulations.

\section{Experiment}

During the stairs exercise experiment, 10 participants' exercise information, including HR and exercise phase (ascending or descending), is collected by a self-designed mobile phone application. In addition, the physiological information is collected by the portable gas analyser-Cosmed $K 4 b^{2}$ (Cosmed, Italy) ${ }^{25}$. The scenario of the experiment, the $K 4 b^{2}$ and the interface of the self-developed mobile phone application are shown in Fig. 1.

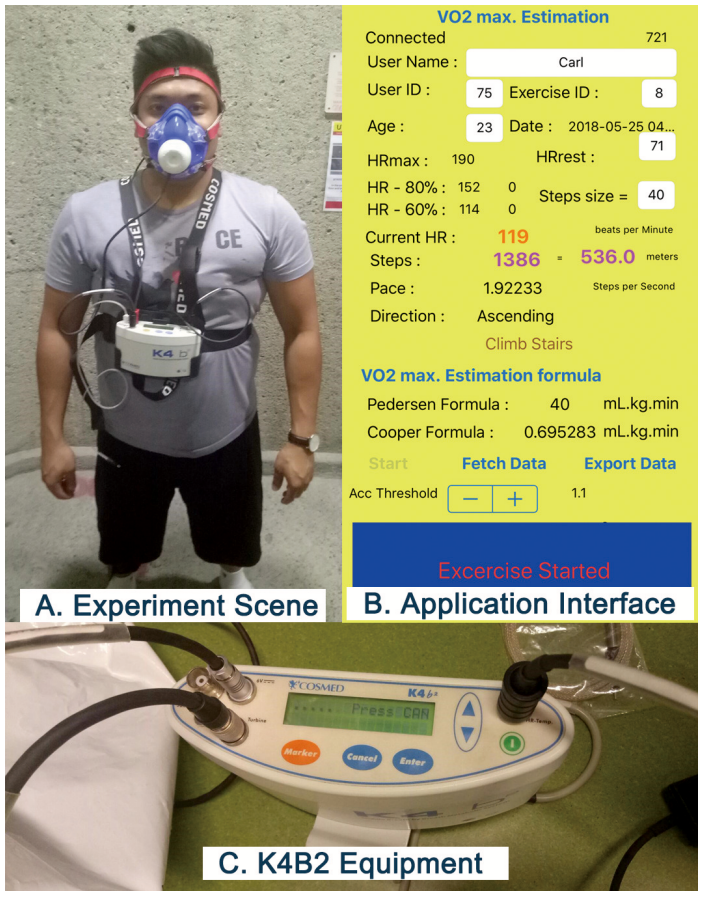

Figure 1. The Scene, Application Interface and Equipment Employed in the Stairs Experiment

Considering the linear relationship between the $\mathrm{HR}$ and $\dot{V} \mathrm{O}_{2}, H R_{\max }{ }^{26277}$ has been recognized as an indicator to detect the 
exercise intensity ${ }^{28}$. One representative formula of $H R_{\max }$ is introduced in Robert's research ${ }^{29}$ as shown in Eq. (1):

$$
H R_{\max }=205.8-0.685 \times \text { age }
$$

where, age indicates the age of the participant.

The mobile phone is placed on the ankle of participants as they go upstairs and downstairs. The exercise phase is acquired from the measurements of the Inertial Measurement Unit (IMU) data. During the exercise, ten untrained (non-athletes) and healthy subjects (no records of motor skill disorder, cardiac-respiratory disorder or related medications history) are instructed to maintain their HR within the range of $60 \%$ to $80 \%$ of their $H R_{\max }$. When their HR starts to exceed $80 \%$ of $H R_{\max }$ during the ascending phase, the participants are instructed to go downstairs. Once their HR is below $60 \%$ of $H R_{\max }$ during the descending phase, the participants are instructed to go upstairs. The range of the HR guarantees that the exercise is in accordance with aerobic circumstance. Meanwhile, the model will not change dramatically and will stay in a linear range. Also, this protocol ensures that all the physiological signals are constantly and randomly changing without the participants feeling uncomfortable. The respiration data, such as $\dot{V} O_{2}$ and $\dot{V} C O_{2}$, are collected by the portable gas analyzer- $K 4 b^{2}$ during the participants' stairs exercise. Each experiment lasts 12 minutes. An entire period during the exercise is defined as from the beginning of the ascending period to the end of the descending period. In this scenario, most participants completed the experiment within two to four periods in around 12 minutes. The statistical physical information of the participants is shown in Table 1.

Table 1. Information about the Study Participants $(n=10)$

\begin{tabular}{|c|c|c|c|c|c|c|}
\hline Information & Age $($ year $)$ & Height $(\mathrm{cm})$ & Mass $(\mathrm{kg})$ & $H R_{\max }$ & $60 \% H R_{\max }$ & $80 \% H R_{\max }$ \\
\hline \hline Mean & 26.2 & 171.2 & 83.6 & 187.8 & 112.6 & 150.2 \\
\hline Standard Deviation & 6.75 & 0.42 & 2.95 & 4.64 & 2.95 & 3.79 \\
\hline
\end{tabular}

The $\dot{V} O_{2}$ is divided by the mass $(\mathrm{kg})$ for each participant to exclude the impact of the participant's mass on breath information. The normalized $\dot{V} O_{2}$ are recorded as $\dot{V}_{d} O_{2}$. The phase of the exercise is described as 2 when the participants are ascending (going upstairs) and as 1 when descending (going downstairs). The phase and measured $\left(\dot{V}_{d} O_{2}\right)$ of one representative participant are shown in Fig. 2.

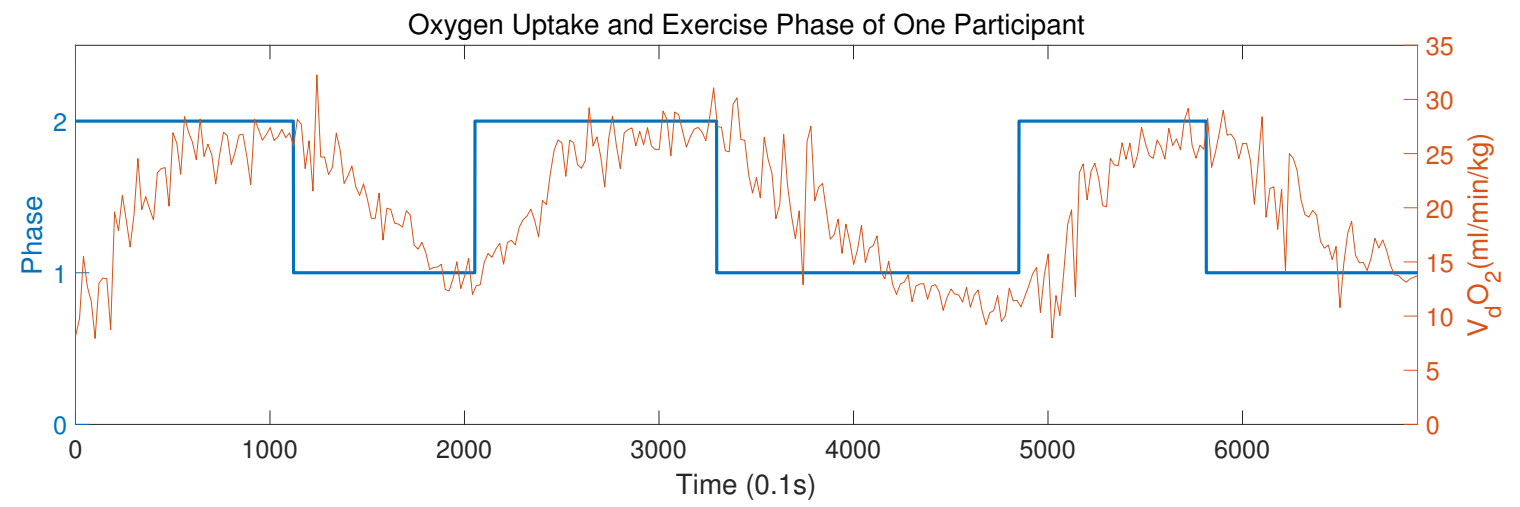

Figure 2. Measured $\dot{V}_{d} O_{2}$ and Exercise Phase of One Participant during the Stairs Exercise

\section{Kernel based Non-parametric Modeling}

A kernel-based non-parametric estimation method is applied to build the phase- $\dot{V} O_{2}$ model. The relationship between the exercise phase (input) and $\dot{V}_{d} O_{2}$ (output) is described as a single input single output (SISO) system. The exercise phase which varies between ascending and descending could be considered as a square input. The output can be calculated by impulse response as Eq. (2):

$$
y(t)=\sum_{\tau=0}^{\infty} u(t-\tau) g[\tau]+\varepsilon(t), t=1,2, \ldots, N,
$$

where $u(t)$ is the input, $y(t)$ is the output, $g(\tau)$ is the impulse response, $t$ is the sampling time, $\varepsilon(t)$ is the Gaussian white noise, and $N$ is the total number of sampling. 
To rewrite Eq. (2) in a compact form, all the elements (row) in $y(t), u(t-\tau)$ and $\varepsilon(t)$ are stacked to form matrices $\boldsymbol{Y}, \boldsymbol{\phi}$ and $\boldsymbol{\varepsilon}$. In addition, we define $\left[\begin{array}{llll}g(0) & g(1) & \cdots & g(m)\end{array}\right]^{T}=\boldsymbol{\theta} \in \mathbb{R}^{m}$, where the vector $\boldsymbol{\theta} \in \mathbb{R}^{m}$ contains the Finite Impulse Response (FIR) coefficients. Then the matrix form of Eq. (2) can be written as an FIR model:

$$
\boldsymbol{Y}=\boldsymbol{\phi} \boldsymbol{\theta}+\boldsymbol{\varepsilon}
$$

The estimation of $\boldsymbol{\theta}$ can be solved by LS estimation as ${ }^{18}$ :

$$
\hat{\boldsymbol{\theta}}=\arg \min _{\boldsymbol{\theta} \in \mathbb{R}^{m}}\|\boldsymbol{Y}-\boldsymbol{\phi} \boldsymbol{\theta}\|^{2}
$$

However, the input of this system is a square signal, and therefore the above equation could be inappropriate for modeling the $\dot{V}_{d} O_{2}$ impulse response (especially when $m$ is big). Often the measurements of $\dot{V}_{d} O_{2}$ contain various artifacts. In order to regularize the estimation and guarantee the validity of the obtained model, we added a regularization term to Eq. (4). This technique could give the structure $\boldsymbol{\theta}$ a "soft" constraint ${ }^{24}$.

$J_{R}(\boldsymbol{\theta})$, which belongs to a Reproducing Kernel Hilbert Space (RKHS) $\mathscr{H}$, is defined as the regularization term:

$$
J_{R}(\boldsymbol{\theta})=\boldsymbol{\theta}^{\mathrm{T}} \boldsymbol{K}^{-1} \boldsymbol{\theta}
$$

where $\boldsymbol{K}$ is a suitable kernel matrix.

Thus, we could get the estimation of $\boldsymbol{\theta}$ :

$$
\hat{\boldsymbol{\theta}}=\arg \min _{\boldsymbol{\theta} \in \mathbb{R}^{m}}\left(\|\boldsymbol{Y}-\boldsymbol{\phi} \boldsymbol{\theta}\|^{2}+\gamma \boldsymbol{\theta}^{\mathrm{T}} \boldsymbol{K}^{-1} \boldsymbol{\theta}\right)
$$

where $\gamma$ is a positive scalar.

Eq. (6) could be adapted as the following description:

$$
\hat{\boldsymbol{\theta}}=\left(\boldsymbol{K} \boldsymbol{\phi}^{\mathrm{T}} \boldsymbol{\phi}+\gamma I_{m}\right)^{-1} \boldsymbol{K} \boldsymbol{\phi}^{\mathrm{T}} \boldsymbol{Y}
$$

where $I_{m} \in \mathbb{R}^{m \times m}$ is an identity matrix with the dimension of $m \times m$.

For the kernel part, we select the Stable Spline (SS) kernel from various kernels to achieve a better performance of the FIR model and a more accurate estimation of $\dot{V}_{d} \mathrm{O}_{2}$ based on previous study ${ }^{3031}$. The SS kernel is described as follows:

$$
\boldsymbol{K}(i, j)=\frac{c}{2} e^{-\beta \min (\mathrm{i}, \mathrm{j})}-\frac{c}{6} e^{-3 \beta \max (\mathrm{i}, \mathrm{j})},
$$

where $c \geq 0,0 \leq \beta<1$.

The parameters $\gamma, c$ and $\beta$ in this identification method need to be well-tuned. The tuning is based on Alessandro and Tianshi's studies ${ }^{2432}$ and we intend to select the best combination of the parameters. The principle of tuning is to find the best fitness between the real $\dot{V}_{d} O_{2}$ and estimated $\dot{V}_{d} O_{2}$ under the premise of ensuring a smooth Impulse Response (IR) and avoiding over-fit. Above all, we choose $\gamma=200, c=1$ and $\beta=0.9985$ after tuning.

\section{Pre-processing, Identification Strategy and Verification}

Due to the individual differences, the total number and length of one entire period are different for each participant. Accordingly, the results of the identification fitness are affected by these factors. Table 2 presents the total number of periods of each participant and provides a summary.

Table 2. Number of Participants in the Various Exercise Periods

\begin{tabular}{|c|c|}
\hline Periods & Number of Participants \\
\hline \hline 2 & 6 \\
\hline 3 & 4 \\
\hline
\end{tabular}


In Table 2, we make a comparison of the fitness of the filtered $\dot{V}_{d} O_{2}$ estimation when the number of periods is $0.5,1,2$, as all the participants have at least two entire exercise periods. This estimation is conducted by the non-parametric model method, which is introduced in Section . After the identification, the Wilcoxon Rank Sum Test is applied to illustrate the significant difference of the IR results with the different number of periods as the results do not follow the normal distribution.

In order to demonstrate the benefit of this method and study model structure of the exercise phase- $\dot{V}_{d} O_{2}$ system, the system identification toolbox in MATLAB is applied to make a comparative identification. The fixed-order model including first, second and third-order are implemented in MATLAB. The goodness of fit of estimated output is calculated by the fit ratio NRMSE (normalised root mean square error):

$$
N R M S E=\left(1-\frac{\left\|\hat{Y}_{N}-Y_{N}\right\|}{\left\|Y_{N}-\left(\overline{Y_{N}}\right)\right\|}\right) .
$$

where $N$ is the total number of sampling, $Y_{N}$ is the real data (reference), $\hat{Y}_{N}$ is the estimated $Y_{N}$ and $\overline{Y_{N}}$ is the mean value of $Y_{N}$.

To verify the changes to the system structure throughout the entire exercise and demonstrate the benefit of the non-parametric model when we focus on the whole exercise period, the IR model and three kinds of fixed-order models identified from the previous period, are applied to estimate the output of the next period for each participant. The goodness of fit is the primary indicator in this stage to study the estimation performance of these approaches.

\section{Results}

This section shows the identification results and the fitness of different models in a different situation. The results concerning the Wilcoxon Rank Sum Test and variance are used to firstly demonstrate the significant difference of the IR from different periods and secondly, to verify the stability of different models. The verification results about the changing structure for different periods are also presented.

Three representative identification results by the non-parametric model for the particular number of periods are shown in Fig. 3, which contains the impulse response and the estimated output $\left(\dot{V}_{d} O_{2}\right)$ from half, one and two periods. The boxplot to describe the fitness results of the non-parametric model over a different number of periods for all participants is presented in Fig. 4.
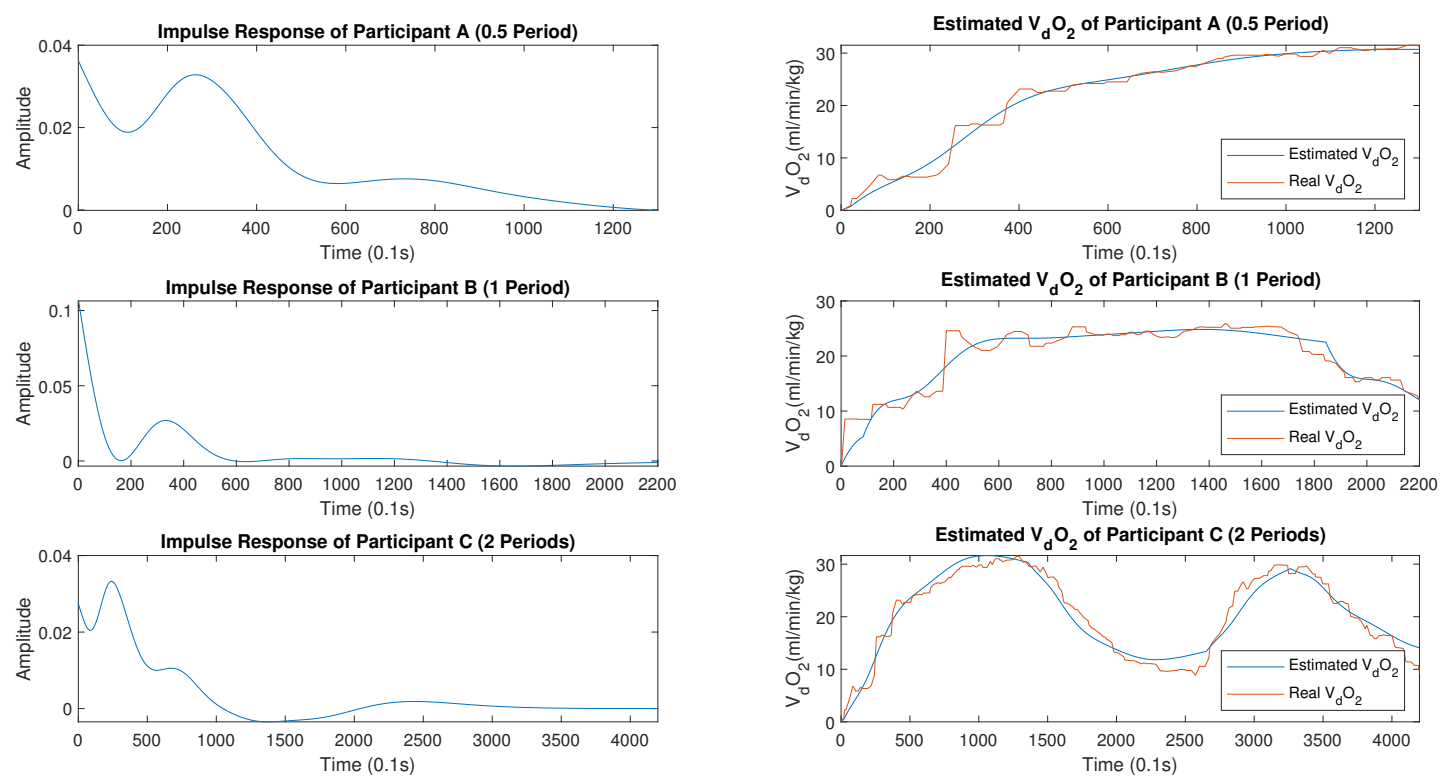

Figure 3. Impulse Response and Estimated $\left(\dot{V}_{d} \mathrm{O}_{2}\right)$ of Three Participants (Half a period, one period and two periods)

The results from one and two periods are highlighted in the next step study as the IR of them ultimately performs at a stable rate as shown in Fig. 3. We calculated the estimated output fitness of 10 participants which are from different methods, including the non-parametric model, and the first-order, second-order and third-order models. The comparison results are 


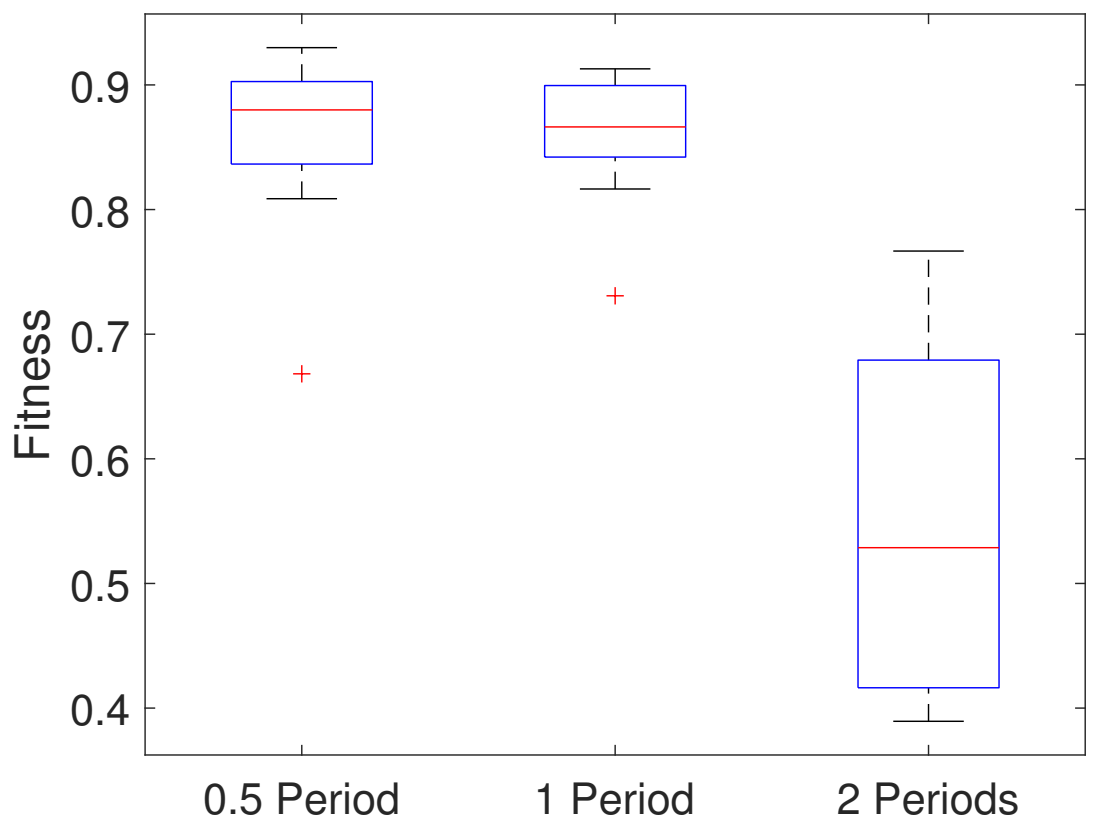

Figure 4. Fitness of Estimated Output of the Non-parametric Model over Different Periods

shown in Fig. 5. The fitness from the four methods of each participant is represented separately. For the second-order and third-order models some results of fitness show a quite concussive or peculiar performance, which means the non-parametric and first-order model is more stable than the higher-order model for this system.
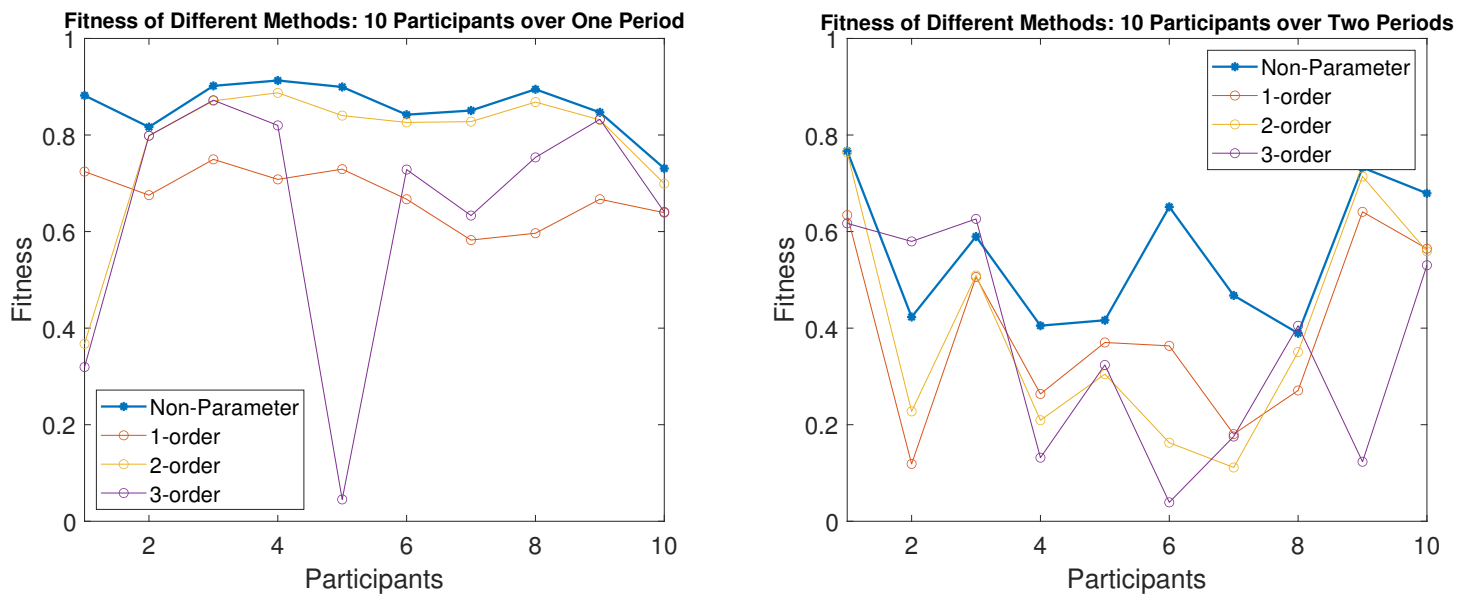

Figure 5. Fitness of Different Methods: 10 Participants over One or Two Periods

The variance of the fitness by different models indicates the stability of the model which is shown in Table 3 . We find that the non-parametric model exhibits the smallest variance in both one period and two periods, which means that this model is more stable and suitable for different situations. Moreover, the first, second and third-order models show different performance as the number of periods change.

In order to demonstrate the significant difference between the different number of periods, the Wilcoxon Rank Sum Test is applied on IR because it does not follow a normal distribution. The IR of the same participants over a different number of periods are compared and the results are shown in Table 4. Generally, $P<0.05$ is considered as statistically significant. All the P value means $h=1$, which also means that all the distinctions of IR (different number of periods with the same participants) are significant. This also illustrates that the system model of a different period is quite different. 
Table 3. Fitness Variance of Different Model Methods over One or Two Periods

\begin{tabular}{|c|c|c|}
\hline Model Type & One Period & Two Periods \\
\hline \hline First-order & 0.0031 & 0.0350 \\
\hline Second-order & 0.0239 & 0.0539 \\
\hline Third-order & 0.0695 & 0.0514 \\
\hline Non-parametric & 0.0030 & 0.0218 \\
\hline
\end{tabular}

Table 4. Rank Sum Test of Same Participants' IR over a Different Number of Periods

\begin{tabular}{|c|c|c|c|}
\hline Participant & Half vs One & One vs Two & Half vs Two \\
\hline \hline 1 & $\mathrm{P}=2.42^{-215}$ & $\mathrm{P}=2.38^{-214}$ & $\mathrm{P}=0$ \\
\hline 2 & $\mathrm{P}=1.73^{-240}$ & $\mathrm{P}=1.11^{-129}$ & $\mathrm{P}=0$ \\
\hline 3 & $\mathrm{P}=5.17^{-113}$ & $\mathrm{P}=4.22^{-258}$ & $\mathrm{P}=0$ \\
\hline 4 & $\mathrm{P}=2.36^{-131}$ & $\mathrm{P}=5.09^{-208}$ & $\mathrm{P}=0$ \\
\hline 5 & $\mathrm{P}=2.26^{-184}$ & $\mathrm{P}=6.31^{-98}$ & $\mathrm{P}=0$ \\
\hline 6 & $\mathrm{P}=1.26^{-214}$ & $\mathrm{P}=2.70^{-110}$ & $\mathrm{P}=0$ \\
\hline 7 & $\mathrm{P}=1.68^{-273}$ & $\mathrm{P}=1.82^{-114}$ & $\mathrm{P}=0$ \\
\hline 8 & $\mathrm{P}=0$ & $\mathrm{P}=7.66^{-20}$ & $\mathrm{P}=0$ \\
\hline 9 & $\mathrm{P}=9.23^{-225}$ & $\mathrm{P}=7.90^{-38}$ & $\mathrm{P}=0$ \\
\hline 10 & $\mathrm{P}=4.90^{-317}$ & $\mathrm{P}=2.14^{-10}$ & $\mathrm{P}=0$ \\
\hline
\end{tabular}

When extending the identification period, the fitness is significantly decreased as shown in Fig. 4 and Fig. 5. We intend to verify the structure of the system is changing with periods and demonstrate that the non-parametric method is more stable. Therefore, the impulse response models acquired from the non-parametric modeling approach and the first-order, second-order, and third-order models in the previous period are implemented to estimate the output in the next period for each participant. The verification fitness of the estimation is shown in Fig. 6.

The initial point of the first period is noted so that it is consistent with the next period when the verification is conducted. According to the results in Fig. 4, Fig. 5 and Table 3, the fitness of the estimation output in the next period declines when using the IR model from the previous model. In addition,, Fig. 6 illustrates that the IR model from the non-parametric model is more robust when the identification period changes. A summary is displayed in Fig. 7 which combines the results from Fig. 5 and Fig. 6. The results of different models (non-parametric model, first-order model, second-order model, third-order model) for different periods (one period, two periods and the previous period model verification for the next period) are directly shown in Fig. 7, and these demonstrate that the non-parametric model performs better than the other three models in different situations.

\section{Discussion}

In this paper, we introduced a non-parametric model to investigate the dynamics of $\dot{V} \mathrm{O}_{2}$ during continuously changing stairs exercises. Our results showed that the performance of the non-parametric model was superior to other fixed-order models, indicating the complexity and variability of the respiratory system in different participants during exercise.

In previous research, the first-order system was proposed for $\dot{V} O_{2}$ estimation during the single onset or offset period experiment ${ }^{113334}$. However, the first-order system is not suitable when the input and output of the system become complicated as in our experiment. The examples of the IR model in Fig. 3 illustrate this conclusion. When the first, second and third-order models are applied in identification, the best fitness for different participants is obtained from different models. Moreover, for some participants, the fitness is quite low when using these three kinds of models. The above aspects illustrate that the system cannot be described as a fixed-order model. In the literature, current $\dot{V} O_{2}$ estimation usually assumes that the physiological process is in a steady-state during exercise, which is just an ideal laboratory contrivance. In fact, humans frequently switch among different metabolic rates when they are awake ${ }^{35}$. As one of the ADL, going upstairs (ascending) is often followed by going downstairs (descending) or vice versa. In addition, physiological signals such as heart rate, respiratory rate, and blood pressure are considered complex and non-linear ${ }^{36373839}$. The advantage of the non-parametric model in system identification under an uncertain system structure has been confirmed in previous studies ${ }^{22324}$. For these reasons, we opted for the ascending and descending phases to provide the input of the non-parametric model. Our fitness analysis shows that the non-parametric model performs well for all of the participants, as shown in Figures 5-7. This indicates that the non-parametric model is suitable for the system when the structure is complicated and changing. The reason for this is that the amount of information is sufficient 


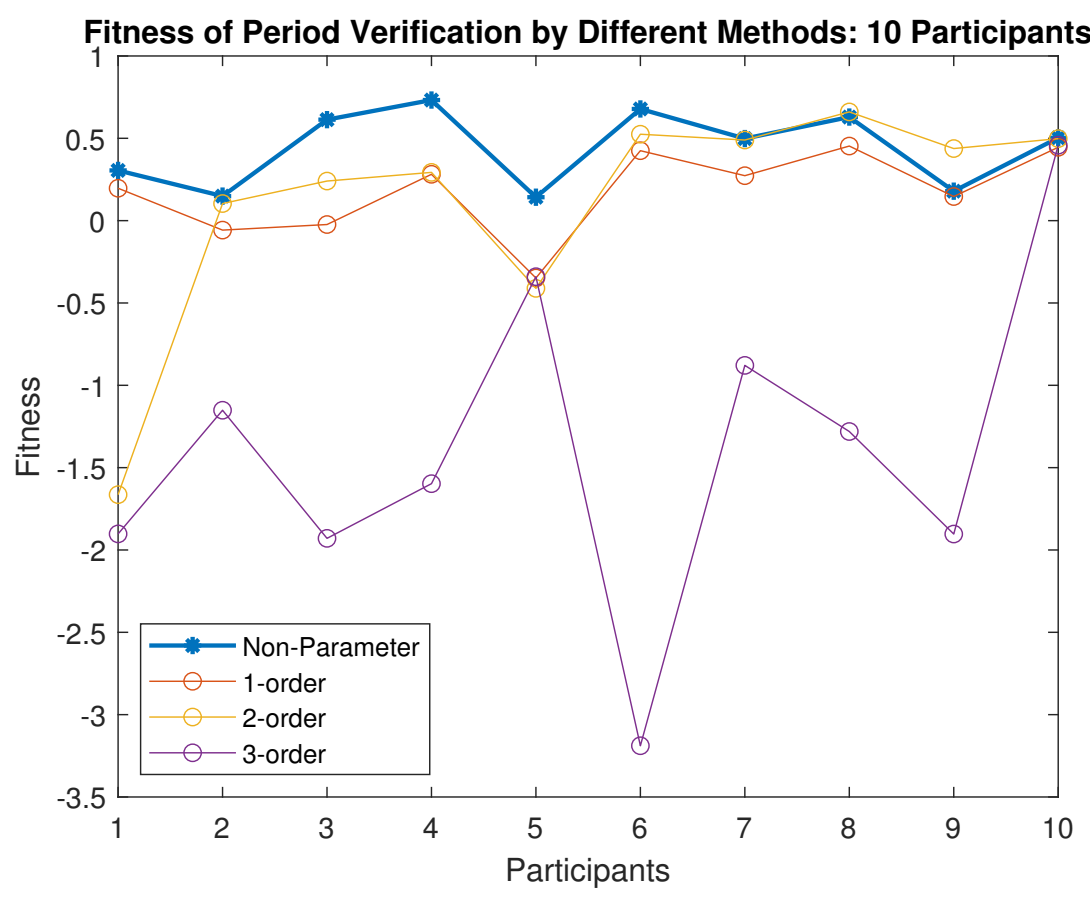

Figure 6. The Fitness of Period Verification by Different Model Methods

when the non-parametric model is applied to fully utilize the prior information for the tuning of the kernel covariance. The prior information in the kernel provides a foundation to estimate the structure of the system and provides the capacity to accommodate the complexity of the model. The input signals from the stairs exercise have significant strength and duration, and the uncertainty of the stochastic input can increase the index of the information matrix. The summarized performance of different models in different periods shown in Fig. 7 also directly and clearly demonstrates the higher fitness of the non-parametric model. It can be concluded that the non-parametric model is more stable and compatible with different participants in different periods.

Our analysis focused on the comparison of different periods since our objective is to investigate the dynamics of $\dot{V} \mathrm{O}_{2}$ during continuously changing stairs exercises. As is evident in Fig. 4, the fitness of two periods is significantly lower than those of half or one period for the non-parametric model. Table 4 illustrates that the IR model is quite different when the number of periods is different. Furthermore, the testing part is conducted to support this point. Comparing Fig. 6 with the left figure in Fig. 5, we can conclude that the IR model from the previous period is not particularly suitable for the next period as the fitness level is lower. In all of the above results, the model of the system changes with different exercise periods. Our experiment requires participants' $H R_{\max }$ to always be maintained in the range of $60 \%$ to $80 \%$. To meet dynamic exercise, the human respiratory system will adjust over time leading to higher complexity. It has been demonstrated that acceleration of pulmonary $\dot{V} O_{2}$ occurred in humans during prolonged periods of training, which is related to enhanced mitochondrial oxidative enzymes activity and increased blood flow ${ }^{40}$. Moreover, the predominant response of $\dot{V} O_{2}$ is driven by contracting muscles during exercise ${ }^{35}$. Therefore, the decrease in fitness with the exercise period may be associated with the dynamic response of the respiratory system and muscle fatigue. Even so, the advantages of the non-parametric model are still obvious in comparison to other IR models. The fitness comparison in Fig. 5 and variance comparison in Table 3 indicate that the non-parametric model is more stable over different numbers of periods. Our experiment protocol guarantees a continuous and random changing $\dot{V} \mathrm{O}_{2}$ during the exercise phase. Combining the complexity and dynamics of the human respiratory system with $\dot{V} \mathrm{O}_{2}$ estimation can facilitate the standardization and development of exercise prescriptions.

\section{Conclusion}

The non-parametric model is applied to investigate the dynamics of $\dot{V} O_{2}$ during stairs exercise. The self-designed application and $K 4 b^{2}$ gas analyzer provide a reliable technique to record $\dot{V} O_{2}$ data and the exercise phase. The protocol of the experiment guarantees a continuous and randomly changing $\dot{V} \mathrm{O}_{2}$ during the exercise phase. The identification results of different numbers of periods are compared to illustrate the variation model of the system. Experimental results indicate that the model from the previous period is no longer suitable for the next period. The fixed-order model includes three types of order which 


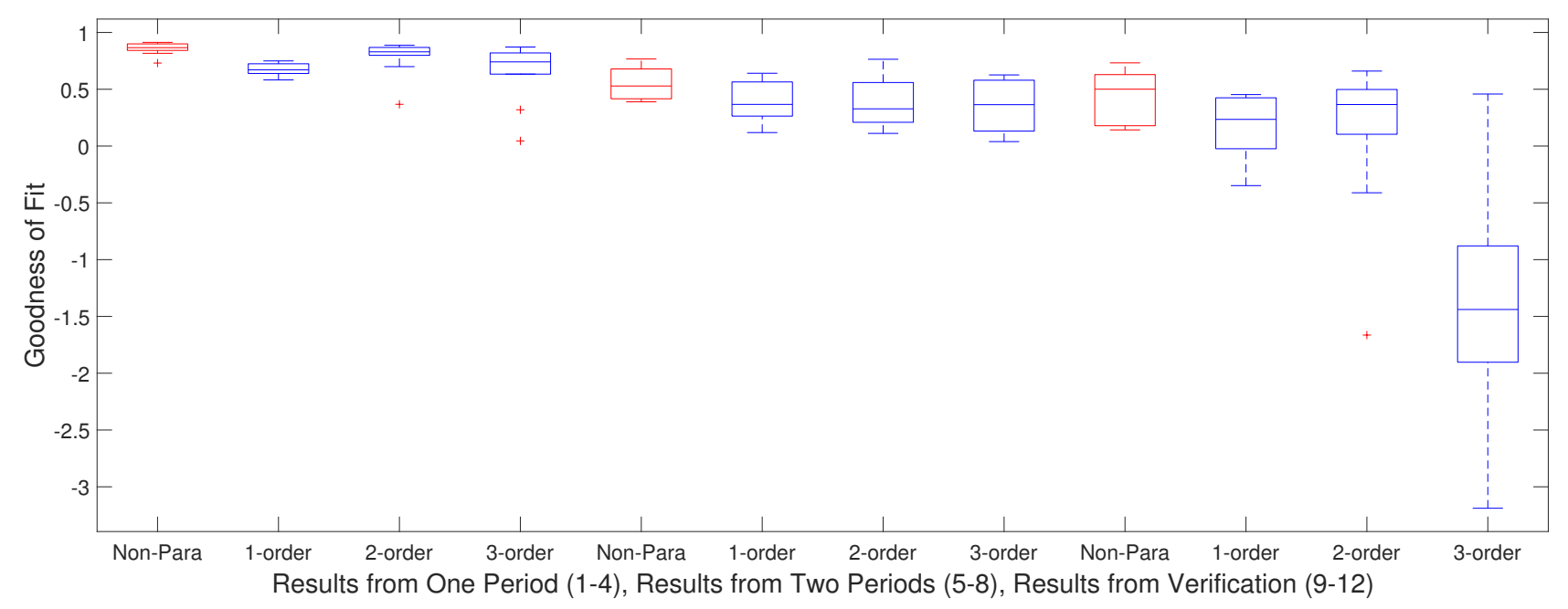

Figure 7. The Boxplot for Fitness of the Different Models over Different Periods

are conducted as comparison approaches to demonstrate the benefits of using the non-parametric modeling approach. It can be concluded that the non-parametric model is more stable and compatible for different participants in different periods. The findings related with respiratory metabolism regulation and muscle fatigue in this research enables us to investigate the conversion mechanism at different metabolic rates of human. The complexity of the human respiratory system, as well as individual differences among subjects, would be worth exploring in more depth in future research.

\section{References}

1. Ilmarinen, J. et al. Training effects of stair-climbing during office hours on female employees. Ergonomics 22, 507-516 (1979).

2. Butts, N., Dodge, C. \& McAlpine, M. Effect of stepping rate on energy costs during stairmaster exercise. Medicine science sports exercise 25, 378-382 (1993).

3. Boreham, C. A., Wallace, W. F. \& Nevill, A. Training effects of accumulated daily stair-climbing exercise in previously sedentary young women. Prev. Medicine 30, 277-281 (2000).

4. Yu, H. et al. Nonparametric model prediction for intelligent regulation of human cardiorespiratory system to prescribed exercise medicine. IEEE Access 8, 224621-224630 (2020).

5. Teh, K. C. \& Aziz, A. R. Heart rate, oxygen uptake, and energy cost of ascending and descending the stairs. Medicine \& Sci. Sports \& Exerc. 34, 695-699 (2002).

6. Cooper, R. A., Fletcher-Shaw, T. L. \& Robertson, R. N. Model reference adaptive control of heart rate during wheelchair ergometry. IEEE transactions on control systems technology 6, 507-514 (1998).

7. Tanaka, H., Monahan, K. D. \& Seals, D. R. Age-predicted maximal heart rate revisited. J. Am. Coll. Cardiol. 37, 153-156 (2001).

8. Loy, S. F. et al. Effects of stairclimbing on vo2max and quadriceps strength in middle-aged females. Medicine science sports exercise 26, 241-247 (1994).

9. Su, S. W. et al. Transient and steady state estimation of human oxygen uptake based on noninvasive portable sensor measurements. Med. \& biological engineering \& computing 47, 1111 (2009).

10. Abe, D., Yanagawa, K. \& Niihata, S. Effects of load carriage, load position, and walking speed on energy cost of walking. Appl. ergonomics 35, 329-335 (2004).

11. Puente-Maestu, L. et al. Effects of two types of training on pulmonary and cardiac responses to moderate exercise in patients with copd. Eur. Respir. J. 15, 1026-1032 (2000).

12. Cooper, R. A. et al. Prediction of pulmonary function in wheelchair users. Spinal Cord 31, 560 (1993). 
13. Gløersen, Ø. N., Gilgien, M., Dysthe, D. K., Malthe-Sørenssen, A. \& Losnegard, T. J. Oxygen demand, uptake, and deficits in elite cross-country skiers during a 15-km race. Medicine \& Sci. Sports \& Exerc. 52, 983-992 (2020).

14. McLaughlin, J., King, G., Howley, E., Bassett Jr, D. \& Ainsworth, B. Validation of the cosmed k4 b2 portable metabolic system. Int. journal sports medicine 22, 280-284 (2001).

15. Duffield, R., Dawson, B., Pinnington, H. \& Wong, P. Accuracy and reliability of a cosmed k4b2 portable gas analysis system. J. Sci. Medicine Sport 7, 11-22 (2004).

16. Altini, M., Penders, J. \& Amft, O. Estimating oxygen uptake during nonsteady-state activities and transitions using wearable sensors. IEEE journal biomedical health informatics 20, 469-475 (2015).

17. Pillonetto, G., Chiuso, A. \& De Nicolao, G. Prediction error identification of linear systems: a nonparametric gaussian regression approach. Automatica 47, 291-305 (2011).

18. Pillonetto, G., Dinuzzo, F., Chen, T., De Nicolao, G. \& Ljung, L. Kernel methods in system identification, machine learning and function estimation: A survey. Automatica 50, 657-682 (2014).

19. Chen, T., Ohlsson, H. \& Ljung, L. On the estimation of transfer functions, regularizations and gaussian processes—revisited. Automatica 48, 1525-1535 (2012).

20. Chen, T., Andersen, M. S., Ljung, L., Chiuso, A. \& Pillonetto, G. System identification via sparse multiple kernel-based regularization using sequential convex optimization techniques. IEEE Transactions on Autom. Control. 59, 2933-2945 (2014).

21. Leeb, H. \& Pötscher, B. M. Model selection and inference: Facts and fiction. Econom. Theory 21, 21-59 (2005).

22. Pillonetto, G. \& De Nicolao, G. A new kernel-based approach for linear system identification. Automatica 46, 81-93 (2010).

23. Chen, T. On kernel design for regularized lti system identification. Automatica 90, 109-122 (2018).

24. Chiuso, A., Chen, T., Ljung, L. \& Pillonetto, G. Regularization strategies for nonparametric system identification. In Decision and Control (CDC), 2013 IEEE 52nd Annual Conference on, 6013-6018 (IEEE, 2013).

25. Pinnington, H. C., Wong, P., Tay, J., Green, D. \& Dawson, B. The level of accuracy and agreement in measures of feo2, feco2 and ve between the cosmed $\mathrm{k} 4 \mathrm{~b} 2$ portable, respiratory gas analysis system and a metabolic cart. J. Sci. Medicine Sport 4, 324-335 (2001).

26. Pollock, M. L. et al. Acsm position stand: the recommended quantity and quality of exercise for developing and maintaining cardiorespiratory and muscular fitness, and flexibility in healthy adults. Med Sci Sports Exerc. 30, 975-991 (1998).

27. Howley, E. T. Type of activity: resistance, aerobic and leisure versus occupational physical activity. Medicine \& Sci. Sports \& Exerc. 33, S364-S369 (2001).

28. Fox III, S. M. \& Skinner, J. S. Physical activity and cardiovascular health. The Am. journal cardiology 14, 731-746 (1964).

29. Robergs, R. A. \& Landwehr, R. The surprising history of the" hrmax $=220$-age" equation. J. Exerc. Physiol. Online 5, $1-10$ (2002).

30. Pillonetto, G. \& De Nicolao, G. Kernel selection in linear system identification part i: A gaussian process perspective. In Decision and Control and European Control Conference (CDC-ECC), 2011 50th IEEE Conference on, 4318-4325 (IEEE, 2011).

31. Chen, T., Ohlsson, H., Goodwin, G. C. \& Ljung, L. Kernel selection in linear system identification part ii: A classical perspective. In Decision and Control and European Control Conference (CDC-ECC), 2011 50th IEEE Conference on, 4326-4331 (IEEE, 2011).

32. Chen, T. \& Ljung, L. Implementation of algorithms for tuning parameters in regularized least squares problems in system identification. Automatica 49, 2213-2220 (2013).

33. Magosso, E. \& Ursino, M. Cardiovascular response to dynamic aerobic exercise: A methematical model. Med. Biol. Eng. Comput. 40, 660-674 (2002).

34. Yu, H. et al. Nonparametric dynamical model of cardiorespiratory responses at the onset and offset of treadmill exercises. Med. \& biological engineering \& computing 1-15 (2018).

35. Poole, D. Towards an understanding of the mechanistic bases of vo_2 kinetics. Oxyg. uptake kinetics sport, exercise medicine (2005). 
36. Caldirola, D., Bellodi, L., Caumo, A., Migliarese, G. \& Perna, G. Approximate entropy of respiratory patterns in panic disorder. Am. J. Psychiatry 161, 79-87 (2004).

37. Hu, X., Miller, C., Vespa, P. \& Bergsneider, M. Adaptive computation of approximate entropy and its application in integrative analysis of irregularity of heart rate variability and intracranial pressure signals. Med. engineering \& physics 30, 631-639 (2008).

38. Xie, H.-B., Chen, W.-T., He, W.-X. \& Liu, H. Complexity analysis of the biomedical signal using fuzzy entropy measurement. Appl. Soft Comput. 11, 2871-2879 (2011).

39. Xie, H.-B., Guo, J.-Y. \& Zheng, Y.-P. Fuzzy approximate entropy analysis of chaotic and natural complex systems: detecting muscle fatigue using electromyography signals. Annals biomedical engineering 38, 1483-1496 (2010).

40. Zoladz, J. A. et al. Mechanisms responsible for the acceleration of pulmonary vo2 on-kinetics in humans after prolonged endurance training. Am. J. Physiol. Integr. Comp. Physiol. 307, R1101-R1114 (2014).

\section{Acknowledgements}

The authors would like to thank Dr. Lin Ye and Dr. Hamzah M Alqudah for the help during this work.

\section{Author contributions statement}

Hairong Yu carried out the detailed theoretical derivation and drafted the manuscript. Tian Xie checked the theoretical derivation and revised the manuscript. Steven Su supervised the theoretical development and revised the manuscript. All authors read and approved the final manuscript.

\section{Additional information}

\section{Competing interests}

The authors declare that they have no competing interests.

\section{Funding}

This work was supported by the National Natural Science Foundation of China (Grant No. 62103449) and the Fundamental Research Funds for the Central Universities (Grant No.2021qntd06).

\section{Ethics approval and consent to participate}

The University of Technology Sydney Human Research Ethics Committee (UTS HREC 2009000227) approved these experiments and informed consent was obtained from all participants before the commencement of data collection.

\section{Informed Consent for publication}

The consent form for publication is signed. 